\title{
AN EXPERIMENTAL STUDY ON THE HEAT ENHANCEMENT AND THE BIO-HEAT TRANSFER USING GOLD MACRO ROD AND ULTRASOUND: A POTENTIAL ALTERNATIVE TO KILL CANCER CELLS
}

\author{
${ }^{1}$ Austerlitz, C., ${ }^{2}$ I. Gkigkitzis, ${ }^{1}$ D. Campos and ${ }^{4}$ I. Haranas \\ ${ }^{1}$ Clínica Diana Campos Recife, PE, BR \\ ${ }^{2}$ Department of Biomedical Physics, East Carolina University, Greenville NC, USA \\ ${ }^{4}$ Research and Development in Electromagnetic Theory Applications at VDG Applications, Toronto, CA
}

Received 2014-03-16; Revised 2014-03-21; Accepted 2014-04-22

\begin{abstract}
We have previously proposed a method of treating solid tumors with a combination of gold macro-rods irradiated with ultrasound. Macro particle sized rods offer a greater circumferential treatment area over nanoparticle options. Experimental studies were conducted to investigate the heat enhancement and the bio-heat transfer to breast chicken using gold macro rod and ultrasound. An ultrasound, other than a focused ultrasound, may produce heat enough to cook a chicken breast up to about $1 \mathrm{~cm}$ diameter if a single gold rod is placed on the superficial tissue. Simulations and experimental results will provide the means to evaluate the treatment, to better design a patient-specific therapy to achieve maximum destruction of tumor and injury minimization of healthy tissue by controlling size, shape and location of gold seeds and ultrasound parameters.
\end{abstract}

Keywords: Gold, Hyperthermia, Ultrasound

\section{INTRODUCTION}

The insufficiency of the response of single Nano spheres to energy sources for the production of controllable hyperthermia and the need to use ensembles of nanoparticles to augment the contribution has been theoretically demonstrated (Fasla et al., 2010). Studies conducted on the NP-induced toxicity have revealed that the metal-based nanoparticles can affect the biological behavior at the organ, tissue, cellular, subcellular and protein levels. The size of the nanoparticles is small and these can easily access the skin, lungs and brain and cause adverse effects (Huster et al., 2007; Lanone et al., 2009).

The use of ultrasound is largely an effort to reduce the use of chemotherapy and radiotherapy for treating cancer. Chemotherapy is considered to impose difficulties because drugs often produce harmful side effects and radiotherapy is also problematic because $\mathrm{x}$-rays travel through normal tissue to arrive at the tumor site and it is known that the $\mathrm{x}$-rays sometimes damage normal tissue. Ultrasound has the ability to noninvasively concentrate energy into a controllable volume deep in tissue (Wu and Nyborg, 2006).

Hyperthermia in cancer treatment has been achieved by magnetic fluid, interstitial microwave probe, long frosted contact probe, magnetic nanoparticles, near infrared-absorbing nanoparticles, gold nanospheres, gold nanorods, gold iron oxide, gold nanoshells, gold coated brass, double doped magnetic silica nanospheres, plasmonic photothermal, non-radioactive ferromagnetic seed interstitial microwave antenna, interstitial laser, radiofrequency, ultrasound, diffuse focus ultrasound, focused ultrasound, laser.

All such methods described so far make use of needles, probes, nanoparticles, optical fibers, ferromagnetic alloys plated with gold. However, there is 
no found in the consulted literature about the use of macro gold rods (which have a relatively high density) irradiated with ultrasound to treat cancer tumor. Experimental studies were conducted to investigate the heat enhancement and the bio-heat transfer to breast chicken using gold macro rod and ultrasound (Fig. 1).

The use of gold rods irradiated with ultrasound to kill cancer cells offers the following advantages as compared to current art. Some advantages are: Cost-compared with irradiation facilities (e.g., gamma knife, cyber knife, linear accelerators, high dose rate brachytherapy and low dose rate brachytherapy), an ultrasound facility is much cheaper. It does not require shielding barriers, high maintenance equipment costs and radiation safety processes, which may include radiation source replacement. Side effects and the seed size-compared with radiotherapy and chemotherapy, the dose therapeutic ratio for ultrasound treatment may be much superior. Besides the short, medium and long term effect of radiation, the accuracy in the absorbed dose delivered to patients treat with radiation should be within $3 \%$. One property of cancerous cells is that they divide rapidly. In chemotherapy this is used by the delivery of drugs that target dividing cells, either causing them to undergo apoptosis or impairing mitosis. Since cancer cells are not the only cells dividing in the body side-effects are common, especially to cells that divide rapidly under normal circumstances such as bone marrow, digestive tract and hair follicles cells. The end point of the heat propagation in tissue for a e.g., $1 \mathrm{~cm}$ length and $0.1 \mathrm{~cm}$ diameter irradiated with ultrasound is about $0.7 \mathrm{~cm}$, when the temperature of the gold seed change for more than $20 \%$. Also, in some cases, normal tissue may be protected from heat by using an appropriate flow of cold water. Nanoparticles are not completely absorbed by the tumor and may be not equal distributed in the tumor. Seed positioning-the gold seed (s) placed in or near the tumor may be checked by means of a CT. This may result in a higher accuracy to irradiate and destroy the target tumor. Number of radioactive seeds-Comparable with prostate cancer treated with $\mathrm{I}-125$ radioactive seeds (apprx. 80 seeds), the treatment with macro gold rods require much less seeds (app. 20 for the mass of tissue). Radiation safety-Compared with HDR, low dose rate brachytherapy or proton therapy, no residual radiation will remain in the patient after the treatment. Simplicitycompared with PDT which depends on the knowledge of the amount of oxygen, photosensitizer and light delivered to the tumor, the non radioactive gold macro rod depends only to the energy delivered to the gold seed by the ultrasound. Besides it does not need toxic substance and require protection against e.g., sun light for a relative long period of time. Compared with radiation treatment, the treatment plan dose may be prescribed without sophisticated computer software. Cosmetic-Compared with surgery and radiation cancer treatment, especial regions of the human body, such as the corner of eyes or nose may not be deformed after the treatment with gold rods. Single treatment-Compared with radiation therapy or chemotherapy which may require several treatment sections, the non-radioactive gold macro seed treatment may be performed at once. Retreatment-Treatment with gold seeds and ultrasound may be redone.

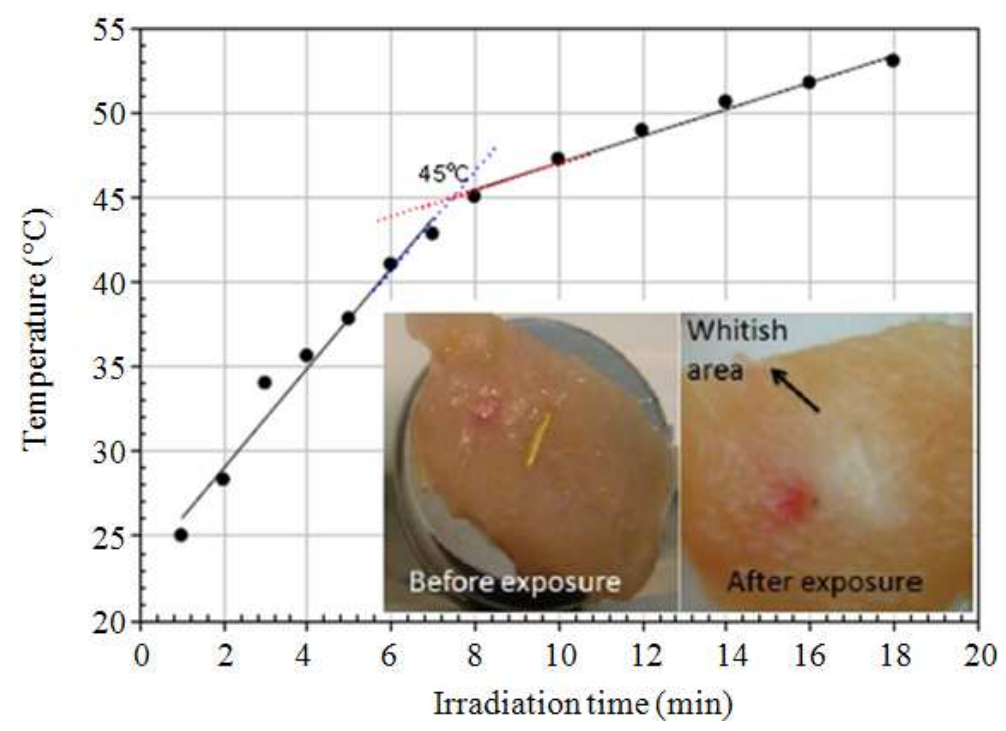

Fig. 1. Temperature enhancement of the gold rod placed on the surface of a $0.5 \mathrm{~cm}$ thick chicken breast irradiated with ultrasound 
Some of the expected disadvantages are: Compared with chemotherapy, the non-radioactive gold macro seed treatment does not cover incipient metastasis targets. Compared with radiation therapy and chemotherapy, the treatment with macro gold rod and ultrasound requires invasive procedure.

\section{MATERIALS AND METHODS}

Chicken breasts: Commercially available fresh chicken breast was used in this study. Ultrasound: An ultrasonic cavitation fast Slimming Equipment Mod. GS8.2E with a medium ultrasound head was used as the irradiation measuring assembly (Guangzhou Cosmetology Equipment Limited Company, Ltd, City/Province: Guangzhou/Guangdong, State: Guizhou, Country: China). Thermometer: An infrared DT-530 thermometer was used to measure the temperature in which the MGR was placed at intervals of $1 \mathrm{~min}$ (Tianjin Cheerman Technology Co., Ltd., City: Tianjin, Province/State: Tianjin, Country/Region: China (Mainland)).

\section{RESULTS}

A $0.5 \mathrm{~cm}$ thick slice of breast chicken with a $1 \mathrm{~cm}$ height and $0.1 \mathrm{~cm}$ diameter gold rod incrusted in its surface was stimulated by ultrasound generated by a transducer oscillating with a nominal frequency of 1 $\mathrm{MHz}$ and power of about $75 \mathrm{~W}$ during a period of time of $18 \mathrm{~min}$ at room temperature. During the irradiation, the temperature of the gold rod was monitored with an infrared thermometer. The amount of heat to breakdown the connective tissue in meat, herein denominate "cook", in the vicinity of the gold rod, as visually seen by the whitish change in color of the meet, was measured with a caliper. The heat enhancement of the gold rod during the irradiation was investigated in a plot of gold rod temperature versus irradiation time.

During the irradiation, the temperature of the gold rod increased from about 28 to $53^{\circ} \mathrm{C}$. The plot, gold rod temperature versus irradiation time (Fig. 1), revealed that the rate of temperature enhancement of the gold rod changes when its temperature reaches about $45^{\circ} \mathrm{C}$. As a first approximation, fitted data between 1 and $8 \mathrm{~min}$ and 10 to $18 \mathrm{~min}$, with a linear correlation coefficient of 0.97 and 0.99 , respectively, have shown that the slope of the first curve overwhelm that of the second curve by a factor of about 3.8. This change in the bio heat transfer has been attributed to changes in the meat properties when it becomes cooked. The radial diameter of the whitish area of the meat around the gold rod was about $1 \mathrm{~cm}$ (Fig. 1). This maximum range of heat transferred from the gold rod to the surrounding tissue agrees with those values predicted by the authors using analytical methods (Gkigkitzis et al., 2012) and measurement performed with infrared camera (Gkigkitzis and Austerlitz, 2012).

\section{DISCUSSION}

Comparisons between experimental measurements and the heat transfer predicted values is available in the literature for models of hyperthermia induced by gold nanoparticle dispersions in different laser energy levels (Huang et al., 2010). Also, the use of ultrasonic energy in heat treating metals and alloys is based on the transmission of elastic vibrations to the parts either through the surrounding medium (water, oil, emulsion, molten metal or salt) or by firm contact with the wave guide. It has been shown that in austenite, the influence of elastic vibrations and the absorption of part of the energy of elastic vibrations leads to a certain increase in the temperature of samples. As it was shown in (Balalaev, 1965), samples may heat up greatly under the influence of ultrasonic vibrations. The passage of ultrasonic waves through a solid body is accompanied by a series of effects such as intense heating. Application of high-power $20 \mathrm{kHz}$ ultrasound resulted in temperature increases of the order of $200^{\circ} \mathrm{C}$ occurring 20 to $30 \mathrm{~s}$ after initiation of insonation in resonant specimens of fine-grained polycrystalline brass, copper and steel (Mignogna and Green, 1981).

Typically, nanoparticles have a size of the order of a few tens of nanometers and heating generated by ultrasound would be limited to the vicinity of the nanoparticle. Thus generally a large number of nanoparticles is needed to effectively heat an entire cell for example, the size of which can be of the order of microns to tens of microns. Complementary use of submicron Silicon Carbide ( $\mathrm{SiC}$ ) particles as photothermal agents for the heating of bacteria by pulsed Mid-Infrared (MIR) radiation has been demonstrated in (Rosenberg and Petrie, 2012) indicating the use of larger size particles for improving the effectiveness of treatment. If a material is a good conductor of heat then the heat will move quickly. Metals are widely used for heat transfer purposes because they have properties which allow for propagation of heat while being able to withstand the temperature extremes sometimes associated with heating.

We have previously calculated the effect of differences in shapes and sizes of gold rod targets irradiated with ultrasound and their heat enhancement and bio-heat transfer in tissue (Gkigkitzis et al., 2012). Rod-shaped gold with height of $1.0 \mathrm{~cm}$ and different diameters were modeled inside an infinity tissue. Computer codes, based on bio-heat diffusion equations, were developed to calculate the temperature elevation in the gold rods and in 
the tissues surrounding it as a result of time exposure and ultrasound power. These theoretical studies indicated that the shape and mass of gold targets irradiated with ultrasound influence the bio-heat transfer in tissue. It was shown that a single $0.1 \mathrm{~cm}$-diameter gold rods implanted in cancer tumor irradiated with ultrasound may be enough to destroy a $1 \mathrm{~cm}$-diameter tumor.

The goal of this study is to further affirm the claim that heat activation through ultrasound on a gold rod implanted into a solid tumor will destroy surrounding tumor tissue. An ultrasound, other than a focused ultrasound, may produce heat enough to cook a chicken breast up to about $1 \mathrm{~cm}$ diameter if a single gold rod is placed on the superficial tissue. The change in color of the cooked tissue has provided a way to study the bio heat effect on tissue irradiated with ultrasound. The change in the value of the rate in the temperature enhancement of tissue irradiated with ultrasound may be used as an indicator between normal tissue and damaged tissue. Thus, analytical considerations about bio-heat transfer in tissue may have to take into account the changes in tissue properties when it is cooked. Further in vitro and in vivo experiments are planned to kill human cancer cell lines and treat induced cancer in mice/rats.

An ultrasound, other than a high intensity, may produce heat enough to cook a cancer tumor up to about $1 \mathrm{~cm}$ diameter if a single gold rod is inserted the tumor. Gold macrorods may be used for identification in ultrasound, CT and X-rays imaging allowing their exact positioning in the target organ or tissue before the ultrasound irradiation. Because the ultrasound beam selectively targets and raises the temperature of the gold rod, this methodology may avoid errors in the irradiation process caused by patient or organ movement. High temperature hyperthermia affects may be attenuated by controlling the size and shape of the gold seeds. Therefore, in clinical setting context and considering small lesions, this method is expected to result in desired dose therapeutic ratios. Gold Macro-Particles (GMPs) and ultrasound (focused or not) leads to accurately energy deliverer to the target tumor.

\section{CONCLUSION}

We have conceived a potential alternative method of treating solid tumors with a combination of heat (ultrasound) and gold macro particle sized rods. Among existing treatment options for solid tumors are surgery, chemotherapy, radiation therapy, photodynamic therapy, cyber knife, brachytherapy and more recently, hypertherapy. Proposed are macroparticle sized gold rods to offer a greater circumferential treatment area over nanoparticle options. Heat transfer with therapeutic ultrasound offers many advantages over and irradiation and other therapies including less toxicity, targeted accuracy, improved efficacy, lower equipment cost and a safer environment for both the patient and the health care provider.

\section{REFERENCES}

Balalaev, G.A., 1965. Zashchita stroitelnykh konstruktsii i khimicheskikh apparatov ot korrozii. Russian.

Fasla, B., R. Benmouna and M. Benmouna, 2010. Modeling of tumor's tissue heating by nanoparticles. J. Applied Phys., 108: 124703. DOI: 10.1063/1.3525089

Gkigkitzis, I., C. Austerlitz and D. Campos, 2012. The effect of the shape and size of gold seeds irradiated with ultrasound on the bio-heat transfer in tissue. Am. J. Clin. Oncol-Canc., 35: 195-6.

Gkigkitzis, I. and C. Austerlitz, 2012. Use of gold macro-rods and ultrasound as a hyperthermia cancer treatment. East Carolina University.

Huang, H.C., K. Rege and J.J. Heys 2010. Spatiotemporal temperature distribution and cancer cell death in response to extracellular hyperthermia induced by gold nanorods. Acs Nano, 4: 2892-2900. DOI: $10.1021 / \mathrm{nn} 901884 \mathrm{~d}$

Huster, D., T.D. Purnat, J.L. Burkhead, M. Ralle and O. Fiehn et al., 2007. High copper selectively alters lipid metabolism and cell cycle machinery in the mouse model of wilson disease. J. Biol. Chem., 282: 8343-55. DOI: 10.1074/jbc.M607496200

Lanone, S., F. Rogerieux, J. Geys, A. Dupont and E. Maillot-Marechal et al., 2009. Comparative toxicity of 24 manufactured nanoparticles in human alveolar epithelial and macrophage cell lines. Part Fibre Toxicol., 6: 1-12. DOI: 10.1186/1743-8977-6-14

Mignogna, R.B. and R.E. Green, 1981. Thermographic investigation of high-power ultrasonic heating in materials. Ultrasonics, 19: 159-160. DOI: 10.1016/0041-624X(81)90095-0

Rosenberg, M. and T.A. Petrie, 2012. Theoretical study on the possible use of $\mathrm{SiC}$ microparticles as photothermal agents for the heating of bacteria. Nanotechnology, 23: 055103-055103. DOI: 10.1088/0957-4484/23/5/055103, PMID: 22238273

Wu, J. and W.L.M. Nyborg, 2006. Emerging Therapeutic Ultrasound. 1st Edn., World Scientific, Hackensack, ISBN-10: 9812566856, pp: 346. 